APS

physics

This is the accepted manuscript made available via CHORUS. The article has been published as:

\title{
Strange Metal in Magic-Angle Graphene with near Planckian Dissipation
}

Yuan Cao, Debanjan Chowdhury, Daniel Rodan-Legrain, Oriol Rubies-Bigorda, Kenji Watanabe, Takashi Taniguchi, T. Senthil, and Pablo Jarillo-Herrero Phys. Rev. Lett. 124, 076801 - Published 18 February 2020 DOI: 10.1103/PhysRevLett.124.076801 


\title{
Strange metal in magic-angle graphene with near Planckian dissipation
}

\author{
Yuan Cao, ${ }^{1, *}$ Debanjan Chowdhury, ${ }^{1,2, *}$ Daniel Rodan-Legrain,${ }^{1}$ Oriol Rubies-Bigorda, ${ }^{1}$ \\ Kenji Watanabe, ${ }^{3}$ Takashi Taniguchi, ${ }^{3}$ T. Senthil, ${ }^{1, \dagger}$ and Pablo Jarillo-Herrero ${ }^{1, \ddagger}$ \\ ${ }^{1}$ Department of Physics, Massachusetts Institute of Technology, Cambridge MA 02139, USA. \\ ${ }^{2}$ Department of Physics, Cornell University, Ithaca NY 14853, USA. \\ ${ }^{3}$ National Institute for Materials Science, \\ Namiki 1-1, Tsukuba, Ibaraki 305-0044, Japan.
}

\begin{abstract}
Recent experiments on magic-angle twisted bilayer graphene have discovered correlated insulating behavior and superconductivity at a fractional filling of an isolated narrow band. Here we show that magic-angle bilayer graphene exhibits another hallmark of strongly correlated systems - a broad regime of $T$-linear resistivity above a small, density-dependent, crossover temperature - for a range of fillings near the correlated insulator. This behavior is reminiscent of similar behavior in other strongly-correlated systems, often denoted 'strange metals, such as cuprates, iron-pnictides, ruthenates and cobaltates, where the observations are at odds with expectations in a weakly interacting Fermi liquid. We also extract a transport "scattering rate", which satisfies a near Planckian form that is universally related to the ratio of $\left(k_{B} T / \hbar\right)$. Our results establish magic angle bilayer graphene as a highly tunable platform to investigate strange metal behavior, which could shed light on this mysterious ubiquitous phase of correlated matter.
\end{abstract}


A panoply of strongly correlated materials have metallic parent states that display properties at odds with the expectations in a conventional Fermi liquid and are marked by the absence of coherent quasiparticle excitations. Some well known families of materials, such as the ruthenates [1, 2], cobaltates [3, 4] and a subset of the iron-based superconductors [5], show non-Fermi liquid (NFL) behavior over a broad intermediate range of temperatures, with a crossover to a conventional Fermi liquid below an emergent low-energy scale, $T_{\text {coh }}$, at which coherent electronic quasiparticles emerge as well-defined excitations. Even more striking examples of NFL behavior are observed in the hole-doped cuprates $[6,7]$ and certain quantum critical heavy-fermion compounds [8] where the incoherent features appear to survive down to the lowest measurable temperatures, i.e. $T_{\text {coh }} \rightarrow 0$, when superconductivity is suppressed externally. In the incoherent regime, all of these materials in spite of being microscopically distinct have a resistivity, $\rho(T) \sim T$, and exhibit a number of anomalous features [8-11] clearly indicating the absence of sharp electronic quasiparticles. In strongly interacting non-quasiparticle systems, it has been conjectured [12] that transport "scattering rates" ( $\Gamma$ ) satisfy a universal 'Planckian' bound $\Gamma \lesssim O\left(k_{B} T / \hbar\right)$ at a temperature $T$. It is however worth noting that in a NFL there is in general no clear definition for $\Gamma$; the scattering rates defined through different measurements, such as dc and optical conductivity, need not be identical [13]. A microscopic basis for Planckian limited scattering in generic electronic models currently does not exist; see however Ref. [14-16] for some attempts. One of the most surprising aspects of incoherent transport in these systems, in spite of these subtleties, is that $\Gamma$ extracted from the dc resistivity (through a procedure specified in Ref. [17]) appears to satisfy a universal form $\Gamma=C k_{B} T / \hbar$ with $C$ a number of order $1[17,18]$.

Recent experiments $[19,20]$ have reported the discovery of a correlation driven insulator at fractional fillings (with respect to a fully filled isolated band) in magic-angle bilayer graphene (MABLG). In MABLG, the relative rotation between two sheets of graphene generates a moiré pattern (Fig. 1a) with a periodicity that is much larger than the underlying interatomic distances in graphene. The theoretically estimated electronic bandwidth, $W$, is strongly renormalized near these small magic-angles [21-23]; then the strength of the typical Coulomb interactions, $U$, becomes at least comparable to (if not greater than) the bandwidth, $U \gtrsim W$. Investigating the properties of MABLG as a function of temperature and carrier density with unprecedented tunability in a controlled setup can lead to new insights into the nature of electronic transport in other lowdimensional strongly correlated metallic systems.

For our experiments, we have fabricated multiple high-quality encapsulated MABLG devices (see Fig. 1a) using the 'tear and stack' technique [24, 25]. As reported earlier [19, 25], we obtain 
band-insulators with a large gap near $n \approx \pm n_{s}$, where $n$ is the carrier density tuned externally by applying a gate voltage and $n_{s}$ corresponds to four electrons per moiré unit cell. On the other hand, correlation driven insulators with much smaller gap-scales $[19,20,26]$ appear near $n \approx \pm n_{s} / 2$; we denote these fillings as $\nu= \pm 2$ from now on (the fully filled band corresponds to $\nu=+4$ ). Doping away from these correlated insulators by an additional amount, $\delta$, with holes $(\nu= \pm 2-\delta)$ and electrons $(\nu= \pm 2+\delta)$ leads to superconductivity (SC) [20, 26]. The superconducting transition temperature $\left(T_{c}\right)$ measured relative to the Fermi-energy $\left(\varepsilon_{F}\right)$, as inferred from low temperature quantum oscillations measurements [20], is high, with the largest value of $\left(T_{c} / \varepsilon_{F}\right) \sim 0.07-0.08$, indicating strong coupling superconductivity [20]. A schematic $\nu-T$ phase diagram for MABLG is shown in Fig.1b.

In this work, we investigate the transport phenomenology of the metallic states in MABLG as a function of increasing temperature over a large range of externally tuned fillings. We have analyzed the temperature dependence of the longitudinal DC resistivity, $\rho(T)$, for a number of devices (MA1 - MA6) over a wide range of fillings, the results of which appear to be qualitatively similar across devices. In order to highlight the universal aspects of the behavior, both within and across different samples, we show the temperature dependent traces of $\rho(T)$ for a range of different $\nu$ in two devices MA1 and MA4 in Fig.1c and Fig.1d respectively. The range of $\nu$ chosen for this purpose is shown as a color-bar in Fig.1b; see caption for details. As it is evident from these traces, at low temperatures the resistivity exhibits highly non-monotonic features as a function of $\nu$, reflecting the complicated and distinct nature of the ground states [27] (see also inset of Fig. 1d). On the other hand, at higher temperatures, the traces for both devices look qualitatively similar and show a clear monotonic trend as a function of the gate-tuned filling in spite of the underlying low-temperature differences. As $\nu$ is tuned externally starting from near the superlattice density, the number of carriers increases resulting in an enhanced conductivity. This simple picture is to be taken seriously only at high temperatures; at low temperatures the effective number of carriers changes non-monotonically across $\nu=-2$ [20]. We identify the characteristic temperature scale associated with the crossover between these distinct regimes, which depends on $\nu$ itself, as $T_{\operatorname{coh}}(\nu)$. For a wide range of fillings in the vicinity of $\nu= \pm 2$, we find that $\rho(T) \sim A T$ for $T \gtrsim T_{\operatorname{coh}}(\nu)$, where $A$ denotes the slope of the resistivity in units of ohms $/ \mathrm{K}(\Omega / \mathrm{K})$. In a given device, $A$ has a relatively weak dependence on $\nu$, but there is a substantial variation in the value of $\mathrm{A}$ for fillings $\nu= \pm 2$ for a given device, as well as in between devices (which have invariably slightly different twist angles, $\theta$ ). As we shall demonstrate below, the linearity of the resistivity is accompanied by a number of puzzling features due to which we refer to this regime as a "strange metal" [28]. 
Fig. 2a shows $\rho(T)$ for two separate MABLG devices, MA3 and MA4, at optimal hole doping, i.e. at fillings where the superconductivity is strongest on the hole-doped side of the correlated insulators at $\nu=-2-\delta$ (MA3) and $\nu=2-\delta$ (MA4). From a linear fit to the regime $T>T_{\text {coh }}$, we obtain $A \approx 335 \Omega / \mathrm{K}$ for MA3 and $A \approx 95 \Omega / \mathrm{K}$ for MA4. It is interesting to note that a naive estimate for the slope of the resistivity, assuming $\rho(T) \sim A T$ and accounting for the spin and valley degeneracies at high temperatures, would lead to a value of $A \sim\left(h / 4 e^{2} W\right)$, up to $O(1)$ prefactors [29]. For an estimated bandwidth of order $W \sim 10 \mathrm{meV}$, this gives $A \approx 60 \Omega / \mathrm{K}$ which is not far from the observed values. We now compare and contrast the above results for MABLG with monolayer graphene (MLG), a chemically similar, but weakly correlated metallic system, that also displays $T$-linear resistivity extending over a much wider range of temperatures. In the inset of Fig. 2a, we show two representative examples of $\rho(T)$ for MLG on a $\mathrm{SiO}_{2}[30]$ and hBN [31] substrate, respectively. The resistivity in MLG devices is approximately two orders of magnitude smaller than MABLG, making it a significantly more conducting metal compared to MABLG. Similarly, the slope of the resistivity for MLG on both substrates is $A \approx 0.1 \Omega / \mathrm{K}$ and is nearly doping independent for a range of different densities near charge neutrality. The slope in MLG is thus approximately three to four orders of magnitude smaller than the corresponding slope in MABLG. When non-twisted bilayer graphene is doped away from charge neutrality, there is almost no observable temperature dependence of the resistivity $[31,32]$ and the typical values of the resistivity are considerably smaller than in MLG at similar densities and temperatures. Thus, a direct comparison between MABLG and non-twisted BLG sheds light on the glaring phenomenological differences between the nature of their transport properties. Since there is some variation in the value of the slopes across different samples, we have studied the dependence of $A$ on the twist angle, $\theta$, as shown in Fig. 2b. The slopes are always evaluated for optimal doping, i.e. the filling at which $T_{c}$ is the highest. Orange (cyan) markers denote fillings with $\nu>0$ $(\nu<0)$, while solid (empty) symbols denote deviations, $\delta>0(\delta<0)$, away from the correlated insulators at $\nu= \pm 2$. As it is clear from the figure, the slopes at a given $\theta$ depend on the sign of $\nu$. Experimentally, it is unclear at what value of $\theta$ the magic-angle condition is satisfied precisely. However, we find the highest superconducting $T_{c}$ around $\theta \sim 1.07^{0}$, which might possibly indicate closest proximity to the magic-angle. While we do not have enough data-points to obtain a clear trend, the typical values of the slopes are large near $\theta=1.07^{0}-1.09^{0}$ (MA5, MA3, respectively) and $\theta=1.16^{0}$ (MA1). Fillings $\nu>0$ also tend to have higher slopes than $\nu<0$. We can also obtain the intercept, $\rho_{0}=\rho(T \rightarrow 0)$, from an extrapolation of the data from high temperatures which gives us a rough estimate of the residual resistivity due to elastic scattering. The results are 
shown in the inset of Fig. $2 \mathrm{~b}$.

One of the most interesting aspects of the low-temperature $(T<30 \mathrm{~K})$ longitudinal transport data in MABLG is that the $T$-linear resistivity is primarily confined to fillings near the correlated insulators at $\nu= \pm 2 \pm \delta$ with $\delta \lesssim 1 / 2$. We note however that we do observe a number of correlated phenomena $[19,20,26,33,34]$, and some unusual transport properties including $T$-linear resistivity, also near $\nu= \pm 1, \pm 3$ (marked by asterisks in Fig.1b) in some of our devices, results that will be reported elsewhere. Fig. 2c shows the traces of $\rho(T)$ in the vicinity of $\nu=-1 / 2$ (see corresponding arrow on the schematic phase diagram in Fig. 1b) for device MA4 ; the traces at $\nu=+1 / 2$ look qualitatively similar. The inset of Fig. 2c shows $\rho(T)$ near charge neutrality at $\nu=0$. It is clear that $\rho(T)$ exhibits qualitatively distinct behavior near these fillings, without any clear indication of a broad regime of $T$-linear resistivity. In Fig.2d and its inset we plot $\rho(T)$ near $\nu=-3 / 2$ and $\nu=+3 / 2$ for device MA4 (fillings marked by arrows in Fig.1b), respectively. While for fillings near $\nu=-3 / 2$ there is no apparent $T$-linear resistivity, there is some indication of such behavior near $\nu=3 / 2$.

One of the most celebrated examples of strange metal behavior is observed in the hole-doped cuprates near optimal doping. It shows a number of remarkable features, including $\rho(T) \sim B T$ over hundreds of kelvin without any apparent signs of crossovers $[6,7,17,18]$, anomalous powerlaw dependent optical conductivities [10] and incoherent spectral functions in the presence of a sharp Fermi surface [9]. Focusing on the transport properties, the typical value of the in-plane sheet resistivity in LSCO (which depends on sample quality) at $T=300 \mathrm{~K}$ is approximately $\rho \sim 0.2-0.3 \mu \Omega \mathrm{cm}[6,7]$, which when normalized by the appropriate interplane distance $(d \sim 6.4$ angstroms) leads to a value of the resistivity, $\rho \sim 3.1 \times 10^{3}-4.7 \times 10^{3} \Omega$. These values are roughly comparable to the measured values of the resistivity in MABLG. On the other hand, the value of the slope in LSCO is approximately $B \sim 1.0-2.0 \mu \Omega \mathrm{cm} / \mathrm{K}[6,7]$, which when normalized by $d$ leads to $B \sim 15.6-31.2 \Omega / \mathrm{K}$. The slope is thus slightly smaller than the typical values in MABLG.

Thus far we have compared the typical (non-universal) values of the resistivities and their slopes across different MABLG devices, as well as with other materials such as MLG and optimally doped cuprates. We now focus on a more detailed presentation and analysis of device MA2. We begin by showing the traces of $\rho(T)$ for MA2 in Fig.3a for a range of $\nu=-2 \pm \delta$, roughly corresponding to the color-bar in Fig.1b. As in the other devices, we observe that the resistivity at high temperatures is linear and it decreases monotonically as $\nu$ increases towards charge neutrality, presumably due to the increase in the number of carriers measured relative to the superlattice filling. We have evaluated the slope $A=d \rho(T) / d T$ at high temperatures for the same range of $\nu$ considered in 
Fig.3a above. Fig.3b shows the values of $A$ as a function of the gate-induced carrier density for fillings near $\nu=-2$. The slope here is obtained directly from a linear least-squares fit to the resistivity [29] for $T>8 \mathrm{~K}$. It is interesting to note that the slope varies non-monotonically with $\nu$. On the other hand, it is possible that a transport "scattering rate" $(\Gamma)$ exhibits more universal character. In order to define and obtain $\Gamma$ from just transport data within the same device, we shall now adopt the procedure used in Ref.[17]. In the regime of $T$-linear resistivity, we write the scattering rate, $\Gamma=C k_{B} T / \hbar$, and define the numerical prefactor $C$ as a function of $\nu$ for device MA2 by assuming that the resistivity can be well described by a Drude-like expression [17, 18], such that

$$
C=\frac{\hbar}{k_{B}} \frac{e^{2} n_{c}(0)}{m^{*}(0)} A,
$$

where $n_{c}(0), m^{*}(0)$ are the measured density and effective mass values at low temperatures, $T \rightarrow 0$. This expression gives an operational definition of $C$, and hence of the scattering rate $\Gamma$. In device MA2, we have also been able to extract the actual low- $T$ carrier density $\left(n_{c}(0)\right)$ and effective mass $\left(m^{*}(0)\right)$ from Shubnikov-de Haas $(\mathrm{SdH})$ quantum oscillations measurements [20, 35]. Note that $n_{c}(0)$, as inferred from $\mathrm{SdH}$ measurements, is not simply proportional to $\nu$ everywhere in the phase-diagram. Instead for $\nu=-2-\delta, n_{c}(0)$ corresponds to the gate-induced density relative to the correlated insulator at $\nu=-2[20]$. On the other hand, for $\nu=-2+\delta, n_{c}(0)$ corresponds to the gate-induced density relative to charge-neutrality at $\nu=0$ [20], in agreement with low-temperature Hall measurements. From the $\mathrm{SdH}$ measurements, we can estimate the Fermi-temperature, $T_{F}=$ $2 \pi \hbar^{2} n_{c}(0) /\left(k_{B} g m^{*}(0)\right)$, where $g$ is the degeneracy factor. Interestingly, $\rho(T)$ does not exhibit any characteristic changes or crossovers as the temperature approaches $T_{F}$ for fillings near $\nu=-2-\delta$, where $T_{F} \lesssim 30 \mathrm{~K}[29]$. In all other examples of strange metals in solid-state systems, the range over which $T$-linear resistivity is observed is significantly below $T_{F}$. MABLG is thus unique in that the $T$-linear resistivity appears to persist to the electron degeneracy temperature, as inferred from the low-temperature measurements.

It is remarkable that $C \sim O(1)$ number for all $\nu$ studied near $\nu=-2$ (see inset of Fig. 3). Moreover, $C$ is weakly dependent on the filling for $\nu=-2-\delta$ and varies from $C \sim 0.2-0.4$. On the other hand, for $\nu=-2+\delta$, the coefficient increases monotonically with increasing $\delta$, with $C \sim 1.0-1.6$. It is interesting to note that the superconducting $T_{c}$ is lower for the fillings where $\Gamma$ is larger [29]. A similar analysis cannot be carried out asymptotically close to the insulating filling $\nu=-2$ because of the absence of low-temperature $\mathrm{SdH}$ measurements of $m^{*}$ and $n_{c}$. However, since the slope in the metallic regime at high temperatures evolves smoothly as a function of $\delta$ 
near $\nu=-2$ and the value of $n_{c} / m^{*}$ is almost independent of the filling, we may naively use the extrapolated value of this ratio for $\delta \rightarrow 0$ to infer that the high-temperature metal at $\nu=-2$ has a similar form of scattering rate.

A similar procedure when used to determine the scattering rates for other strongly correlated metals $[17,18]$, including ones that lead to unconventional superconductivity at low temperatures, also leads to $C \sim O(1)$ number. A major advantage of our analysis for MABLG is that all of our measurements are carried out on the same device (MA2) to extract $C$, as opposed to using data from multiple devices at different dopings. For various members of the cuprate family, the coefficient $C \sim 0.7-1.2$, both on the hole and electron-doped side of the phase-diagram $[17,18]$. It is worth mentioning that $C \sim O(1)$ number even for metals like copper, aluminum and palladium above their respective Debye temperatures, where they exhibit a $T$-linear resistivity due to electronphonon scattering [17]. In MLG, the $T$-linear resistivity is also likely due to electron-phonon scattering above a density-dependent temperature [36]. We can calculate the scattering rates for MLG on $\mathrm{SiO}_{2}$ in the regime where they display $T$-linear resistivity. Let us express the resistivity (in units of $h / e^{2}$ ) as

$$
\rho(T)=\frac{h}{e^{2}} \frac{\hbar \Gamma}{2 \varepsilon_{F}(V)},
$$

where $\Gamma$ is as defined earlier and $\varepsilon_{F}(V)$ is controlled by an external gate-voltage, $V$. This leads to an approximate estimate of $C \sim 0.009-0.022$ for the entire range of densities studied in Ref. [30], which is a few orders of magnitude smaller than the value in MABLG. In Table I, we show a few representative examples of systems where the scattering rate has been calculated using the above procedure.

We now critically examine some possible mechanisms that may be responsible for the unusual transport phenomenology in MABLG. Recently, different electron-phonon scattering models have been suggested trying to explain the origin of $T$-linear resistivity in twisted BLG near the magicangle [37-39]. These theoretical models are applicable primarily in the vicinity of fillings $\nu=0$, for angles close to (but not including) the magic angle, and the coefficient of the high temperature $\rho \sim T$ regime is then controlled by the strongly renormalized band-structure near charge-neutrality (Dirac-points). However, in our devices, clear evidence for $\rho \sim T$ and Planckian scattering rates can only be seen near the commensurate fillings of $\nu= \pm 2 \pm \delta$ with $\delta \lesssim 1 / 2$ for $T<30 K$, but not in the vicinity of charge neutrality $|\nu| \lesssim 1 / 2$. Similarly, a recent transport study of twisted BLG devices at angles $\theta \gtrsim 2^{0}[40]$ has reported a power-law behavior of the resistivity, $\rho(T) \sim \rho_{0}+T^{\beta}$, with a non-monotonic value of $\beta \sim 0.9-1.7$ as a function of filling. As in our case, super-linear 
behavior (power law exponent substantially higher than 1) was observed near charge neutrality. A non-linear behavior of the resistivity has also been seen very recently near charge neutrality for devices with angles between $1^{0}$ and $2^{0}$ [41]. Moreover, our data show that $T_{\text {coh }}(\nu)$ can be as low as $0.5 \mathrm{~K}$ (e.g. for device MA4 near $\nu=-2$ in Fig.2a), which is an order of magnitude lower than the predicted crossover temperature above which linear behavior is expected[37]. The maximum value of the slope of the $T$-linear resistivity, which has a weak dependence on $\nu$ in a given device, is observed near the angle $\left(\theta \sim 1.07^{0}\right)$ as well as at an angle of $\theta \sim 1.16^{0}$ (Fig.2b). However, refs.[3739] predict a very strong angle dependence of the slope, with the largest value near the magic-angle, and this dependence is tied to the strong renormalization of the Dirac velocity. Although we do not observe this trend, such a discrepancy could arise as a result of long-wavelength disorder and slow variations in the twist-angle across our devices. In addition, these models seem to underestimate the slope values and their angular dependence substantially, possibly indicating that phonons may not represent the full contribution to the observed $T$-linear behavior. A more sophisticated future theory of electron-phonon scattering that incorporates: i) the underlying details of the electronic band structure of MABLG away from charge-neutrality, including the strongly non-linear energy momentum dispersion and presence of van-Hove singularities, as well as ii) a microscopic treatment of the electron-phonon 'gauge' coupling $[42,43]$ and a possible renormalization of the deformation potential due to electron-electron interactions, iii) a folding of the phonon dispersion due to the moiré brillouin-zone, as well as iv) the possible effects of density and twist-angle disorder, would be highly desirable for a detailed comparison to our experimental data. However, we also note that since the $T$-linear resistivity persists to scales $T \sim T_{F}$ without any signs of a crossover near $\nu=-2-\delta$, while the electrons are no longer in the degenerate limit, electron-phonon scattering in the metallic state $\left(T>T_{c}\right)$ might still be insufficient to explain the origin of these unusual results. In principle, $T$-linear resistivity can arise as a result of scattering off any electronic collective mode fluctuations (instead of phonons) for $T_{M} \lesssim T$, where $T_{M}$ is a characteristic scale associated with the respective collective mode, but a crossover across $T \sim T_{F}$ is nevertheless expected. It is unclear at the moment if such electronic collective modes are present near $\nu= \pm 2$.

Under conditions of local equilibrium, the conductivity is given by $\sigma=\chi D_{c}$, where $\chi=\partial n / \partial \mu$ is the electronic compressibility and $D_{c} \sim v^{2} / \Gamma$ is the charge diffusivity $(v \equiv$ a characteristic velocity). Thus far, we have inherently assumed that the temperature dependence of the resistivity arises as a result of the temperature dependence of $\Gamma \sim k_{B} T / \hbar$. An alternative scenario that could, in principle, lead to $\rho \sim T$ at high temperatures arises solely from the temperature dependence of the compressibility, $\chi \sim 1 / T$, in a system with a bounded kinetic energy. However, in some of 
our devices the $\rho \sim A T$ behavior persists from high temperatures down to temperatures as low as $0.5 \mathrm{~K}$ without any change in the slope, $A$ (e.g. device MA4 in Fig. 2a). Moreover, as emphasized earlier, $\rho$ does not show any crossovers as the temperature is swept through the estimated $\varepsilon_{F}$ for fillings near $\nu=-2-\delta$. Thus while it is unlikely that the above mechanism is responsible for the observed temperature dependence of the resistivity, a systematic future study of the charge compressibility in MABLG as a function of increasing temperature is highly desirable. Instead of relying on the dc transport measurements to extract $\Gamma$, it will be interesting to measure and analyze the extent to which the optical scattering rates in MABLG satisfy a universal form in future experiments. Interestingly, recent measurements of diffusivity and compressibility in a coldatoms based experiment [44] find $\rho \sim T$ from temperatures higher than the bandwidth down to lower temperatures without any signs of a crossover.

In light of these results, any successful theory has to account for the following universal aspects of the phenomenology: (i) a $T$-linear resistivity with values $O\left(h / e^{2}\right)$ in the vicinity of commensurate fillings, $\nu= \pm 2$, with near Planckian $(C \sim O(1))$ "scattering rates", (ii) a weak dependence of the slope of the resistivity on $\nu$, (iii) relative insensitivity of the resistivity to $T_{F}$ and by extension, to the underlying details of the Fermi-surface at low temperatures, and (iv) the presence of a small $T_{\text {coh }}$ above which the transport is unconventional. Since $T_{\text {coh }}$ can be as low as $0.5 \mathrm{~K}$ in some of our devices, it will be interesting to see if future experiments find evidence of $T$-linear resistivity down to even lower temperatures (i.e. $T_{\text {coh }} \rightarrow 0$ ). If so, it is possible that the NFL behavior in MABLG is controlled by a $T=0$ quantum critical point or quantum critical phase; there is not enough conclusive experimental information available at present to elaborate on this aspect. On the other hand, if $T_{\text {coh }}$ is non-zero, it is likely that the metallic regime of MABLG for fillings near $\nu= \pm 2$ realizes an intermediate-scale NFL. Assuming that the temperature remains smaller than the typical interaction strengths but large compared to $T_{\mathrm{coh}}$, the state is neither a classical liquid (or gas) nor a degenerate quantum liquid. The system can then be best described as a "semi-quantum" liquid, with no coherent quasiparticle excitations and no sharply defined Fermi surface. Describing such a regime in a theoretically controlled limit is challenging but recent progress has been made in the study of some models $[13,45]$ which find evidence of such incoherent behavior. Guided by these studies and by the present experiments we will further develop microscopic theories of transport in such a regime elsewhere.

We acknowledge Helena Briones for the assistance on the figures for the device structure. This work has been primarily supported by the National Science Foundation (DMR-1809802), the Center for Integrated Quantum Materials under NSF grant DMR-1231319, and the Gordon and Betty 
Moore Foundation's EPiQS Initiative through Grant GBMF4541 to P.J.-H. for device fabrication, transport measurements, and data analysis. This work was performed in part at the Harvard University Center for Nanoscale Systems (CNS), a member of the National Nanotechnology Coordinated Infrastructure Network (NNCI), which is supported by the National Science Foundation under NSF ECCS award no. 1541959. D.C. was supported by a fellowship from the Gordon and Betty Moore Foundation, under the EPiQS initiative, grant GBMF4303, at MIT. D.R.-L acknowledges support from Fundació Bancaria "la Caixa" (LCF/BQ/AN15/10380011) and from the US Army Research Office grant no. W911NF-17-S-0001. O.R.-B acknowledges support from Fundació Privada Cellex. T.S. is supported by a US Department of Energy grant DE-SC0008739, and in part by a Simons Investigator award from the Simons Foundation. K.W. and T.T. acknowledge support from the Elemental Strategy Initiative conducted by the MEXT, Japan, A3 Foresight by JSPS and the CREST (JPMJCR15F3), JST. 


\begin{tabular}{|c|c|c|c|c|}
\hline & Material & \begin{tabular}{|} 
Slope of resistivity $(A)$ \\
3D: $\mu \Omega \mathrm{cm} / \mathrm{K}$ \\
$2 \mathrm{D}: \Omega / \mathrm{K}$
\end{tabular} & $C$ & Refs. \\
\hline \multirow{6}{*}{$3 \mathrm{D}$} & $\mathrm{CeCoIn}_{5}$ & 1.6 & 1 & {$[17]$} \\
\hline & $\mathrm{CeRu}_{2} \mathrm{Si}_{2}$ & 0.91 & 1.1 & {$[17]$} \\
\hline & $(\mathrm{TMTSF})_{2} \mathrm{PF}_{6}(11.8 \mathrm{kbar})$ & 0.38 & 0.9 & {$[17]$} \\
\hline & $\mathrm{UPt}_{3}$ & 1.1 & 1.1 & {$[17]$} \\
\hline & $\mathrm{Cu}(T>100 \mathrm{~K})$ & $7 \times 10^{-3}$ & 1.0 & {$[17]$} \\
\hline & $\mathrm{Au}(T>100 \mathrm{~K})$ & $8.4 \times 10^{-3}$ & 0.96 & {$[17]$} \\
\hline \multirow{5}{*}{ (Quasi-)2D } & $\operatorname{Bi} 2212(p=0.22)$ & 8.0 & 1.1 & {$[18]$} \\
\hline & $\operatorname{LSCO}(p=0.26)$ & 8.2 & 0.9 & {$[18]$} \\
\hline & $\operatorname{PCCO}(x=0.17)$ & 1.7 & 1.0 & {$[18]$} \\
\hline & MLG on $\mathrm{SiO}_{2}$ & 0.1 & $0.01-0.02$ & [30], This work \\
\hline & MABLG & $100-300$ & $0.2-1.6$ & This work \\
\hline
\end{tabular}

TABLE I. Materials exhibiting Planckian scattering rates $(\Gamma)$, where $\Gamma=C k_{B} T / \hbar$. 


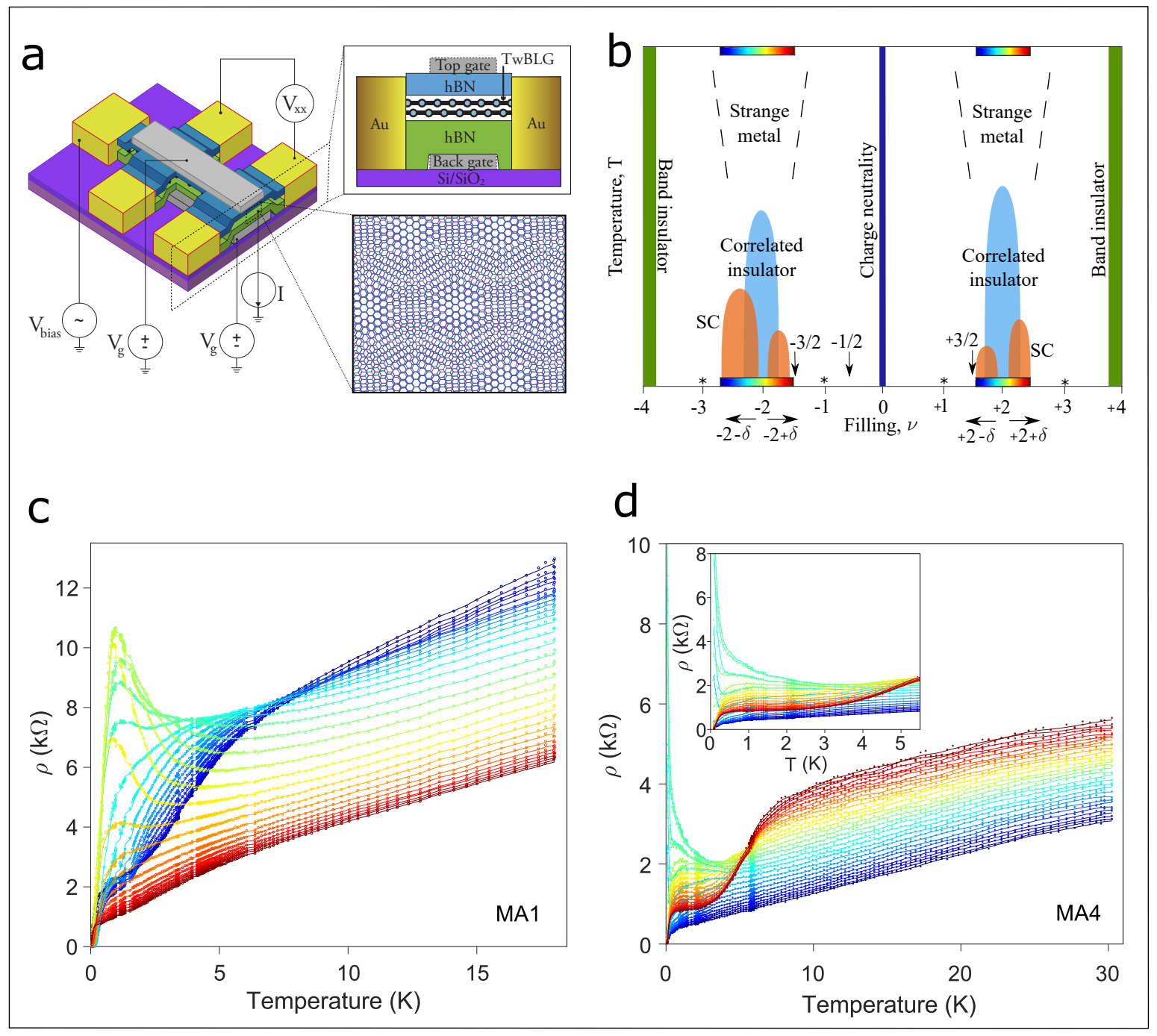

FIG. 1. Transport in MABLG. a, Device schematic and four-probe transport measurement configuration (left). Top-right: side-view of the device structure, consisting of bilayers of graphene twisted by $\theta$ relative to each other, sandwiched by hBN on the top and bottom. The carrier density is tuned using the metal gates at the top/bottom. Bottom-right: schematic MABLG moiré pattern. b, A schematic phase diagram for MABLG as a function of temperature and filling, $\nu$. The strange metal behavior with $T$-linear resistivity is primarily restricted to fillings near $\nu= \pm 2$. Orange regions denote superconductivity. Light blue regions denote the correlated insulator region. The color bars indicate the approximate filling ranges investigated and shown in fig. 1c,d. c, Resistivity $(\rho)$ as a function of temperature for device MA1 $\left(\theta=1.16^{0}\right)$ for gateinduced densities from $-1.96 \times 10^{12} \mathrm{~cm}^{-2}$ (blue) to $-1.22 \times 10^{12} \mathrm{~cm}^{-2}$ (red); see horizontal colorbar near $\nu=-2$ in Fig.1b. d, $\rho(T)$ for device MA4 $\left(\theta=1.18^{0}\right)$ for gate-induced densities ranging from $1.23 \times 10^{12}$ $\mathrm{cm}^{-2}$ (blue) to $1.89 \times 10^{12} \mathrm{~cm}^{-2}$ (red); see horizontal colorbar near $\nu=+2$ in Fig.1b. The inset shows the traces for the same device at low temperatures. The solid smooth lines have been obtained by using a gaussian-weighted filter. 


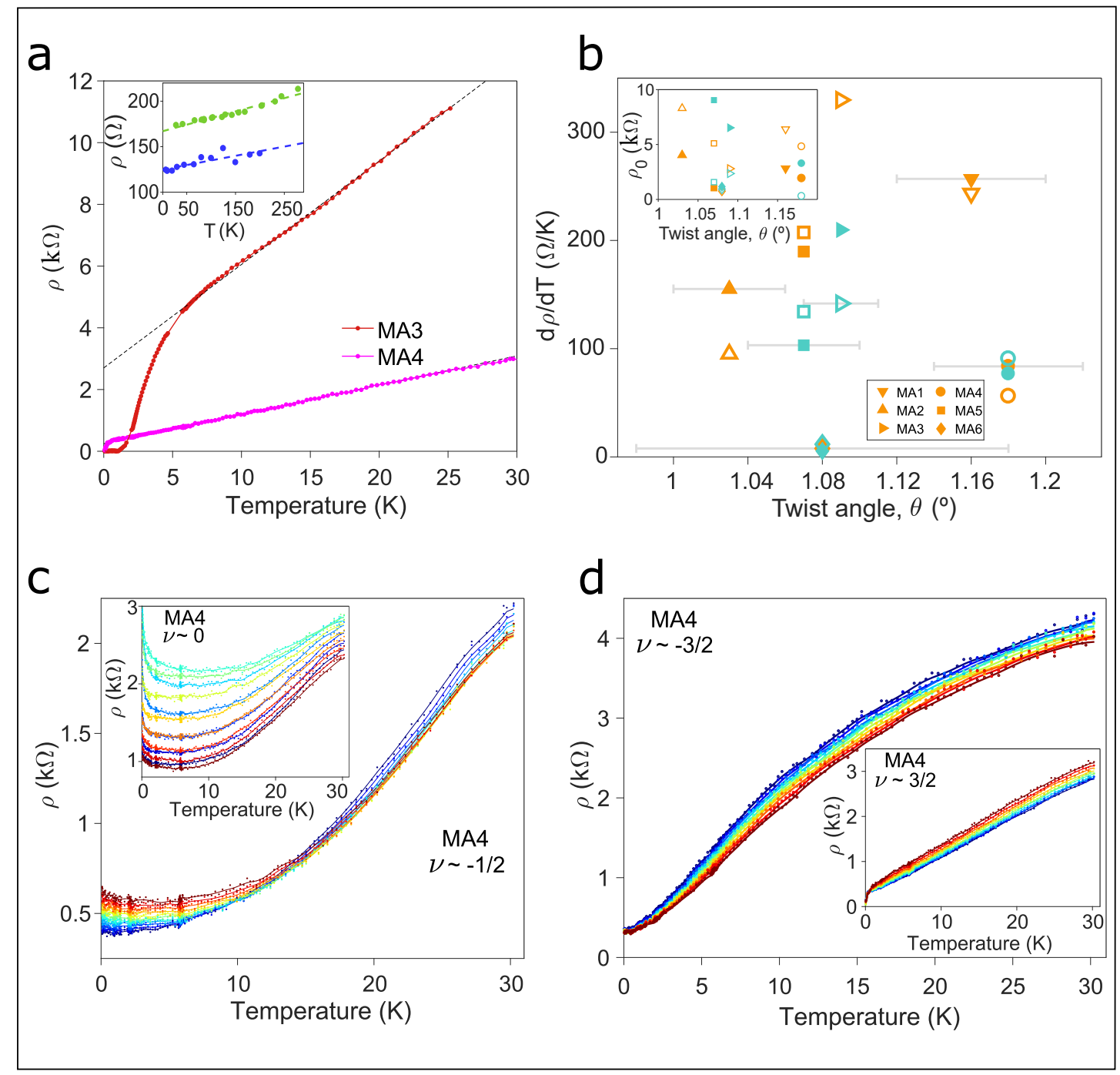

FIG. 2. $T$-linear resistivity near $\nu= \pm 2$ in MABLG. a, Resistivity $(\rho)$ as a function of temperature for device MA3 $\left(\theta=1.09^{0}\right)$ and MA4 $\left(\theta=1.18^{0}\right)$ at a gate-tuned density of $-1.46 \times 10^{12} \mathrm{~cm}^{-2}(\nu=-2-\delta)$ and $1.19 \times 10^{12} \mathrm{~cm}^{-2}(\nu=2-\delta)$, respectively. The inset shows $\rho(T)$ in ohms for MLG on $\mathrm{SiO}_{2}$ (green) and hBN (blue), respectively (data from $[30,31]$ ). $\mathbf{b}$, The slopes $A=d \rho / d T$ obtained at the fillings near $\nu= \pm 2 \pm \delta$ with optimal superconducting $T_{c}$ for six different devices (MA1 - MA6) as a function of respective twist-angles. Orange (cyan) markers denote fillings with $\nu>0(\nu<0)$, while solid (empty) symbols denote deviations, $\delta>0(\delta<0)$, away from the correlated insulators at $\nu= \pm 2$. The inset shows the extrapolated resistivity, $\rho_{0}$, for the same devices. c, $\rho(T)$ for device MA4 near $\nu=-1 / 2$ for gate-induced densities $-0.51 \times 10^{12} \mathrm{~cm}^{-2}$ (blue) to $-0.29 \times 10^{12} \mathrm{~cm}^{-2}$ (red). The data look similar near $\nu=+1 / 2$. The inset shows $\rho(T)$ for the same device near $\nu=0$ and densities between $-0.13 \times 10^{12} \mathrm{~cm}^{-2}$ (blue) to $0.13 \times 10^{12}$ $\mathrm{cm}^{-2}$ (red). $\mathbf{d}, \rho(T)$ for device MA4 near $\nu=-3 / 2$ for gate-induced densities $-1.28 \times 10^{12} \mathrm{~cm}^{-2}$ (blue) to $-1.08 \times 10^{12} \mathrm{~cm}^{-2}$ (red). The inset shows $\rho(T)$ for device MA4 near $\nu=+3 / 2$ from $1.08 \times 10^{12} \mathrm{~cm}^{-2}$ (blue) to $1.28 \times 10^{12} \mathrm{~cm}^{-2}$ (red). 

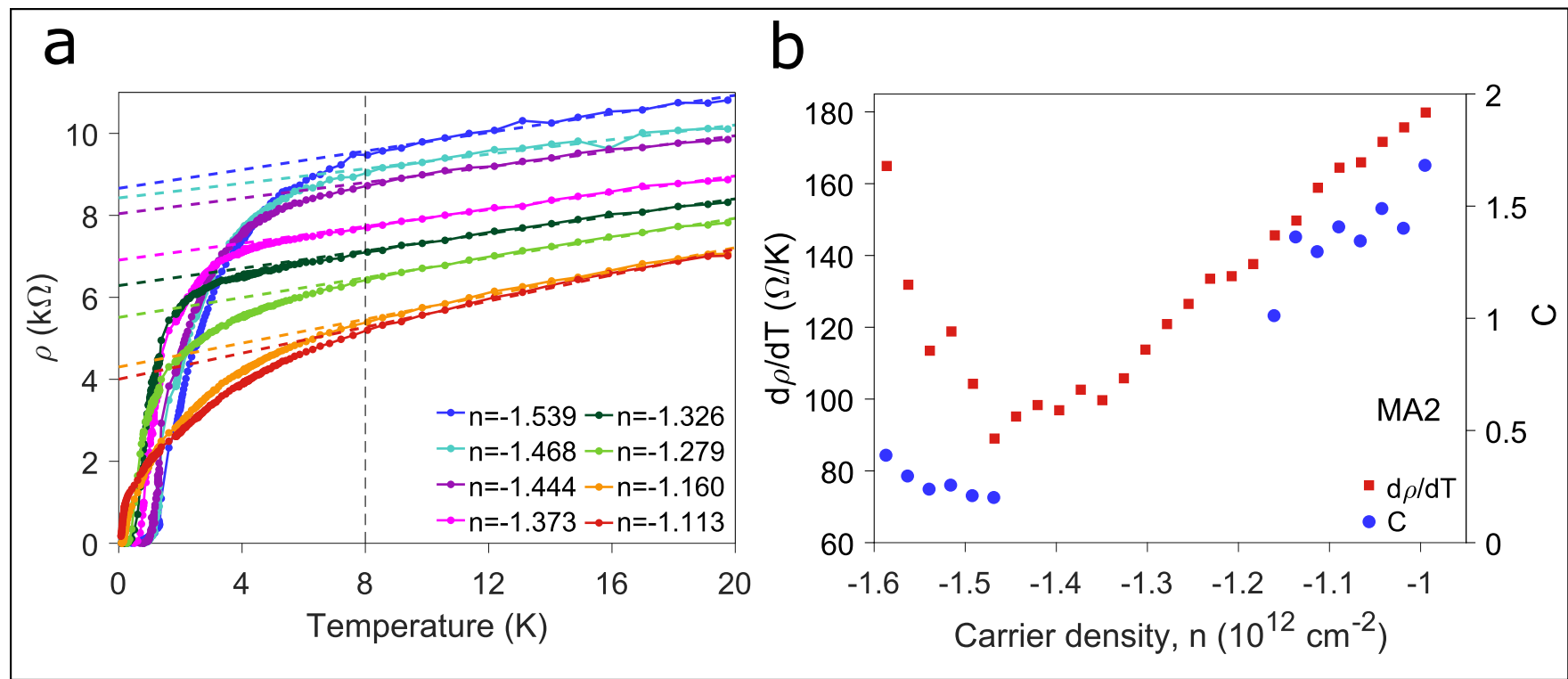

FIG. 3. Planckian scattering in magic-angle bilayer graphene. a, Resistivity as a function of temperature for device MA2 $\left(\theta=1.03^{0}\right)$ near $\nu=-2$ for gate-tuned densities $-1.539 \times 10^{12} \mathrm{~cm}^{-2}$ (blue) to $-1.113 \times 10^{12} \mathrm{~cm}^{-2}$ (red). The correlated insulator at $\nu=-2$ is approximately located near $-1.3 \times 10^{12}$ $\mathrm{cm}^{-2}$. The solid smooth lines have been obtained by using a gaussian-weighted filter. $\mathbf{b}$, Left axis: Slope of resistivity as a function of filling above $8 \mathrm{~K}$ for the same device evaluated using a linear fit. Right axis: The coefficient $C$ of the scattering rate $\Gamma=C k_{B} T / \hbar$ for the same range of fillings. 
* These authors contributed equally

$\dagger$ senthil@mit.edu

$\ddagger$ pjarillo@mit.edu

[1] N. E. Hussey, A. P. Mackenzie, J. R. Cooper, Y. Maeno, S. Nishizaki, and T. Fujita, Phys. Rev. B 57, 5505 (1998).

[2] L. Klein, J. S. Dodge, C. H. Ahn, G. J. Snyder, T. H. Geballe, M. R. Beasley, and A. Kapitulnik, Phys. Rev. Lett. 77, 2774 (1996).

[3] S. Y. Li, L. Taillefer, D. G. Hawthorn, M. A. Tanatar, J. Paglione, M. Sutherland, R. W. Hill, C. H. Wang, and X. H. Chen, Phys. Rev. Lett. 93, 056401 (2004).

[4] Y. Wang, N. S. Rogado, R. J. Cava, and N. P. Ong, Nature 423, 425 (2003).

[5] T. Shibauchi, A. Carrington, and Y. Matsuda, Annual Review of Condensed Matter Physics 5, 113 (2014).

[6] H. Takagi, B. Batlogg, H. L. Kao, J. Kwo, R. J. Cava, J. J. Krajewski, and W. F. Peck, Phys. Rev. Lett. 69, 2975 (1992).

[7] P. Giraldo-Gallo, J. A. Galvis, Z. Stegen, K. A. Modic, F. F. Balakirev, J. B. Betts, X. Lian, C. Moir, S. C. Riggs, J. Wu, A. T. Bollinger, X. He, I. Božović, B. J. Ramshaw, R. D. McDonald, G. S. Boebinger, and A. Shekhter, Science 361, 479 (2018).

[8] H. v. Löhneysen, A. Rosch, M. Vojta, and P. Wölfle, Rev. Mod. Phys. 79, 1015 (2007).

[9] A. Damascelli, Z. Hussain, and Z.-X. Shen, Rev. Mod. Phys. 75, 473 (2003).

[10] D. v. d. Marel, H. J. A. Molegraaf, J. Zaanen, Z. Nussinov, F. Carbone, A. Damascelli, H. Eisaki, M. Greven, P. H. Kes, and M. Li, Nature 425, 271 (2003).

[11] S.-C. Wang, H.-B. Yang, A. K. P. Sekharan, H. Ding, J. R. Engelbrecht, X. Dai, Z. Wang, A. Kaminski, T. Valla, T. Kidd, A. V. Fedorov, and P. D. Johnson, Phys. Rev. Lett. 92, 137002 (2004).

[12] J. Zaanen, Nature 430, 512 (2004).

[13] D. Chowdhury, Y. Werman, E. Berg, and T. Senthil, Phys. Rev. X 8, 031024 (2018).

[14] M. Blake, Phys. Rev. Lett. 117, 091601 (2016).

[15] T. Hartman, S. A. Hartnoll, and R. Mahajan, Phys. Rev. Lett. 119, 141601 (2017).

[16] A. A. Patel and S. Sachdev, Phys. Rev. Lett. 123, 066601 (2019).

[17] J. A. N. Bruin, H. Sakai, R. S. Perry, and A. P. Mackenzie, Science 339, 804 (2013).

[18] A. Legros, S. Benhabib, W. Tabis, F. Laliberté, M. Dion, M. Lizaire, B. Vignolle, D. Vignolles, H. Raffy, Z. Z. Li, P. Auban-Senzier, N. Doiron-Leyraud, P. Fournier, D. Colson, L. Taillefer, and C. Proust, Nature Physics 14 (2018), 10.1038/s41567-018-0334-2.

[19] Y. Cao, V. Fatemi, A. Demir, S. Fang, S. L. Tomarken, J. Y. Luo, J. D. Sanchez-Yamagishi, K. Watanabe, T. Taniguchi, E. Kaxiras, R. C. Ashoori, and P. Jarillo-Herrero, Nature 556, 80 (2018).

[20] Y. Cao, V. Fatemi, S. Fang, K. Watanabe, T. Taniguchi, E. Kaxiras, and P. Jarillo-Herrero, Nature 
556, 43 (2018).

[21] J. M. B. Lopes dos Santos, N. M. R. Peres, and A. H. Castro Neto, Phys. Rev. Lett. 99, 256802 (2007).

[22] E. Suárez Morell, J. D. Correa, P. Vargas, M. Pacheco, and Z. Barticevic, Phys. Rev. B 82, 121407 (2010).

[23] R. Bistritzer and A. H. MacDonald, Proceedings of the National Academy of Sciences 108, 12233 (2011).

[24] K. Kim, A. DaSilva, S. Huang, B. Fallahazad, S. Larentis, T. Taniguchi, K. Watanabe, B. J. LeRoy, A. H. MacDonald, and E. Tutuc, Proceedings of the National Academy of Sciences 114, 3364 (2017).

[25] Y. Cao, J. Y. Luo, V. Fatemi, S. Fang, J. D. Sanchez-Yamagishi, K. Watanabe, T. Taniguchi, E. Kaxiras, and P. Jarillo-Herrero, Phys. Rev. Lett. 117, 116804 (2016).

[26] M. Yankowitz, S. Chen, H. Polshyn, Y. Zhang, K. Watanabe, T. Taniguchi, D. Graf, A. F. Young, and C. R. Dean, Science 363, 1059 (2019), http://science.sciencemag.org/content/363/6431/1059.full.pdf.

[27] Interestingly for both devices at fillings near $\nu=-2-\delta$ (MA1) and $\nu=2+\delta$ (MA4), we observe a broad shoulder around $5 \mathrm{~K}$ where the resistivity drops by a substantial fraction. The suppression in the resistivity, before the eventual onset of SC, could be a signature of strong local pairing fluctuations. We will elaborate on this elsewhere.

[28] The term "strange metal" is typically used to denote a wide variety of unusual metallic behavior, seemingly at odds with the expectations in a weakly interacting Fermi liquid. All strange metals do not necessarily have identical reasons for the strangeness. Our usage of the term is in keeping with this practice.

[29] See supplementary information for more details, which includes Refs. [46-48].

[30] J.-H. Chen, C. Jang, S. Xiao, M. Ishigami, and M. S. Fuhrer, Nature Nanotechnology 3, 206 (2008).

[31] C. R. Dean, A. F. Young, I. Meric, C. Lee, L. Wang, S. Sorgenfrei, K. Watanabe, T. Taniguchi, P. Kim, K. L. Shepard, and J. Hone, Nature Nanotechnology 5, 722 (2010).

[32] S. V. Morozov, K. S. Novoselov, M. I. Katsnelson, F. Schedin, D. C. Elias, J. A. Jaszczak, and A. K. Geim, Phys. Rev. Lett. 100, 016602 (2008).

[33] A. L. Sharpe, E. J. Fox, A. W. Barnard, J. Finney, K. Watanabe, T. Taniguchi, M. A. Kastner, and D. Goldhaber-Gordon, arXiv e-prints , arXiv:1901.03520 (2019), arXiv:1901.03520 [cond-mat.mes-hall].

[34] X. Lu, P. Stepanov, W. Yang, M. Xie, M. A. Aamir, I. Das, C. Urgell, K. Watanabe, T. Taniguchi, G. Zhang, A. Bachtold, A. H. MacDonald, and D. K. Efetov, arXiv e-prints , arXiv:1903.06513 (2019), arXiv:1903.06513 [cond-mat.str-el].

[35] In principle, the effective mass that enters transport quantities can be different from the effective cyclotron mass, as deduced from quantum oscillations measurements.

[36] S. Das Sarma, S. Adam, E. H. Hwang, and E. Rossi, Rev. Mod. Phys. 83, 407 (2011).

[37] F. Wu, E. Hwang, and S. Das Sarma, arXiv e-prints , arXiv:1811.04920 (2018), arXiv:1811.04920 [cond-mat.mes-hall].

[38] I. Yudhistira, N. Chakraborty, G. Sharma, D. Y. H. Ho, E. Laksono, O. P. Sushkov, G. Vignale, and 
S. Adam, Phys. Rev. B 99, 140302 (2019).

[39] H. Ochoa, arXiv e-prints, arXiv:1905.10850 (2019), arXiv:1905.10850 [cond-mat.mes-hall].

[40] T.-F. Chung, Y. Xu, and Y. P. Chen, Phys. Rev. B 98, 035425 (2018).

[41] H. Polshyn, M. Yankowitz, S. Chen, Y. Zhang, K. Watanabe, T. Taniguchi, C. R. Dean, and A. F. Young, arXiv e-prints , arXiv:1902.00763 (2019), arXiv:1902.00763 [cond-mat.str-el].

[42] T. Sohier, M. Calandra, C.-H. Park, N. Bonini, N. Marzari, and F. Mauri, Phys. Rev. B 90, 125414 (2014).

[43] C.-H. Park, N. Bonini, T. Sohier, G. Samsonidze, B. Kozinsky, M. Calandra, F. Mauri, and N. Marzari, Nano Letters 14, 1113 (2014).

[44] P. T. Brown, D. Mitra, E. Guardado-Sanchez, R. Nourafkan, A. Reymbaut, C.-D. Hébert, S. Bergeron, A. M. S. Tremblay, J. Kokalj, D. A. Huse, P. Schauss, and W. S. Bakr, arXiv e-prints , arXiv:1802.09456 (2018), arXiv:1802.09456 [cond-mat.quant-gas].

[45] C. H. Mousatov, I. Esterlis, and S. A. Hartnoll, arXiv e-prints , arXiv:1803.08054 (2018), arXiv:1803.08054 [cond-mat.str-el].

[46] J. Orenstein, G. A. Thomas, A. J. Millis, S. L. Cooper, D. H. Rapkine, T. Timusk, L. F. Schneemeyer, and J. V. Waszczak, Phys. Rev. B 42, 6342 (1990).

[47] C. C. Homes, S. V. Dordevic, M. Strongin, D. A. Bonn, R. Liang, W. N. Hardy, S. Komiya, Y. Ando, G. Yu, N. Kaneko, X. Zhao, M. Greven, D. N. Basov, and T. Timusk, Nature 430, 539 (2004).

[48] T. Hazra, N. Verma, and M. Randeria, arXiv e-prints , arXiv:1811.12428 (2018), arXiv:1811.12428 [cond-mat.supr-con]. 